\title{
Conocimientos sobre el climaterio y sus consecuencias en Obstetras que acuden a capacitarse en Psicoprofilaxis del Climaterio
}

\author{
Nieves Bedón Cortéz'
}

\section{RESUMEN}

El objetivo principal del estudio fue establecer el nivel de conocimiento sobre el climaterio y sus consecuencias en Obstetras de diversas Regiones de Costa y Sierra del Perú. Fueron un total de 170 profesionales que voluntariamente Ilenaron una encuesta que valoró: conocimiento, actitudes y prácticas en la atención ambulatoria. Todas las encuestadas fueron muj eres, sólo el $20 \%$ había recibido capacitación previa y en EsSalud. No hubo diferencia significativa entre el tiempo de egreso y el nivel de conocimiento, pero sí en la disposición y manejo de terapia hormonal por las más jóvenes. Siendo muy significativo el nivel de falta de conocimiento tanto en el profesional de la costa como de la sierra. El 100\% estuvo de acuerdo en la necesidad de capacitarse en esta etapa de la vida. (Horiz Med 2014; 14(1): 49-53)

Palabras clave: Climaterio, nivel de conocimiento, consejería, Psicoprofilaxis del climaterio. (Fuente: DeCS BIREME).

Knowledge of menopause and its consequences in Midwifes attending training in Climacteric Psychoprophylaxis

\begin{abstract}
The main objective of the study was to establish the level of knowledge about menopause and its consequences in midwifes both Coast and Mountain region sof Peru. Were a total of 170 professionals who voluntarily completed a survey that assessed: knowledge, attitudes and practices in ambulatory care. All respondents were women, only $20 \%$ had received prior training in EsSalud. There was no significant difference between the time of discharge and the level of knowledge, but in the provision and management of hormone therapy for younger women. Being very significant level of lack of knowledge in both the professional and the coast mountain range. $100 \%$ agreed on the need for training in this stage of life.
\end{abstract}

(Horiz Med 2014; 14(1): 49-53)

Key words: Climacteric,level of knowledge, counseling, climacteric psychoprophylaxis. (Source: MeSH NLM).

${ }^{1}$ Obstetra, Magister en Gerencia de Servicios de Salud 


\section{INTRODUCCIÓN}

Las proyecciones de población muestran que el Perú, al 30 de junio del 2013 superó los 30 millones 475 mil habitantes, y se ubica como el quinto país con más población de América Latina. Siendo la población de mujeres superior al de los hombres al llegar a los 45 años (50.2\%) y superior al $51.5 \%$ al llegar a los 60 años (1).

Situación que se hace más evidente al tener un incremento de la esperanza de vida y observar que cada vez habrán más mujeres que vivirán su climaterio. Etapa de la vida que transcurre desde la fase reproductiva a la no reproductiva (2).

Hoy, diferentes medios de comunicación divulgan el valor de la prevención y la necesidad de hacer promoción de la salud, sin embrago; todavía se siguen confundiendo términos y existe una gran promoción de productos "naturales" y alternativas para aliviar los síntomas vasomotores y vegetativos al llegar la menopausia (3).

Temas que valdrían la pena analizar y discutir.

El profesional de la Obstetricia, como parte de su formación académica aprende y desarrolla la construcción del conocimiento acerca de los cambios de la mujer en cada etapa de su vida. Con énfasis en los cambios que ocurren en la mujer al cesar su función reproductiva: Psicoprofilaxis del Climaterio (4).

La demanda y necesidad de contar con profesionales de la salud capacitados para atender con calidad a la mujer durante su climaterio cada vez será mayor, motivo por el cual todos los involucrados: estado, universidades y sociedad deberíamos tomar conciencia y desarrollar estrategias que soporten la gran carga social que tendrá nuestro país en los próximos años.

Especialmente, cuando hasta el momento no existe un Plan Nacional del Climaterio.

El objetivo del presente trabajo fue conocer el nivel de conocimientos que tienen las Obstetras de diversas Regiones del Perú, acerca del climaterio y sus consecuencias dado el incremento de consulta por mujeres mayores de 45 años.

\section{EL ESTUDIO}

Se realizó un estudio descriptivo, observacional y transversal sobre el nivel de conocimientos de las Obstetras de Costa y Sierra del Perú, acerca del climaterio y sus consecuencias.

Todas acudieron a un Taller de Capacitación de Psicoprofilaxis del Climaterio y aceptaron participar voluntariamente.

Llenaron una encuesta de 20 preguntas que valoró el nivel de conocimiento, actitud y prácticas sobre consejería y uso de terapia hormonal en pacientes climatéricas que acuden a la consulta ambulatoria en el sector público y privado.

Ingresaron al estudio profesionales de Costa y Sierra del Perú: Piura, Lambayeque, Huancayo y Arequipa, entre enero y noviembre del 2013.

Esto permitió diferenciar y comparar a las encuestadas por edad, tiempo de egreso, capacitación previa y uso o no de terapia hormonal. Asimismo, se pudo comparar el nivel de conocimiento si su trabajo lo desarrollaba con pacientes de la Costa o Sierra.

Los datos se colocaron en una ficha especialmente diseñada para ser analizadas luego de procesados con frecuencias y porcentajes, en una hoja de cálculo en Excel 2010.

El estudio fue revisado y la encuesta aprobada por un grupo de expertos en Climaterio.

\section{HALLAZGOS}

En total, participaron 170 Obstetras que completaron la encuesta de valoración del nivel de conocimientos acerca de climaterio y sus consecuencias. 88 pertenecían al sector privado (51.76\%) y 82 al sector público.

Siendo proporcional el número de profesionales que laboraban en la Costa (Piura y Lambayeque) y en la 
Sierra, a más de 2,500 metros sobre el nivel del mar (Huancayo y Arequipa), 84 y 86 respectivamente. Con relación a la capacitación sobre climaterio, luego de egresar; 34 (20\%) de todas las participantes, recibieron algún tipo de información.

Siendo de EsSalud el $100 \%$ de las capacitadas.

Del grupo que trabajaba en el sector privado, la mayoría tenían menos de 5 años de egresadas, $54(61.36 \%)$ a diferencia de las que laboran en el sector público, $50(60.97 \%)$ que tenían más de 10 años de egreso.

De manera general, tanto las profesionales que laboraban en el sector privado como público, independientemente de su Región, no tenían experiencia de recetar terapia hormonal $71.59 \%$ y $70.73 \%$ respectivamente.

Con relación al nivel de conocimiento, 24 (14.11\%) con menos de 5 años de egresada tuvieron un nivel de conocimiento malo y 58 (34.11\%) de las egresadas con más de 10 años, presentando diferencia estadísticamente significativa.

Lo que se evidenció al tener buen nivel de conocimiento el $\mathbf{2 8 . 2 3 \%}$ de las egresadas con menos de 5 años, con respecto a 13 (7.64\%) de las que tenían más de 10 años de egresadas. Tabla 1.

La mayoría de las encuestadas 84 (49.41\%), independientemente de su Región de trabajo tuvieron un nivel malo de conocimientos (con una $p \varangle 0.001)$.

No hubo diferencia significativa en la indicación de la terapia hormonal tanto a nivel del mar como por encima de los 2,500 metros sobre el nivel del mar.

Siendo muy significativa $(p<0.001)$ el que no indicarían hormonoterapia, 71.42\% y 75.58\% respectivamente. Tabla 2.

Todas consideraron que era necesario capacitarse y organizar un Programa de Psicoprofilaxis del Climaterio, como parte de la atención de la mujer que Ilega a su climaterio.
Tabla 1. Nivel de conocimiento sobre el climaterio en relación a tiempo de egreso

$\begin{array}{cccccccc}\begin{array}{c}\text { Nivel de } \\ \text { Conocimientos }\end{array} & \text { Malo } & \text { Regular } & \text { Bueno } & \mathbf{p} \\ \text { Tiempo de egresada } & \mathrm{n} & \% & \mathrm{n} & \% & \mathrm{n} & \% & \\ \text { menos de 5 años } & 24 & 14.11 & 10 & 0.58 & 48 & 28.23 & <0.005 \\ \text { 5 a 10 años } & 2 & 1.17 & 5 & 2.9 & 1 & 0.58 & \\ \text { más de 10 años } & 58 & 34.11 & 9 & 5.21 & 13 & 7.64 & \\ \text { Total } & 84 & 49.41 & 24 & 14.11 & 62 & 36.47 & \end{array}$

Tabla 2. Indicación de TRH

$\begin{array}{cccccc}\begin{array}{c}\text { Nivel de } \\ \text { Conocimientos }\end{array} & \text { A nivel del mar } & \text { más de } 2500 \mathrm{msnm} & \mathbf{p} \\ \text { Indicación de TRH } & \mathrm{n} & \% & \mathbf{n} & \% & \\ \text { SI } & 24 & 28.57 & 21 & 24.41 & <0.001 \\ \text { NO } & 60 & 71.42 & 65 & 75.58 & \\ \text { TOTAL } & 84 & 100 & 86 & 100\end{array}$

\section{DISCUSIÓN}

La menopausia, causada por la disminución de la función ovárica debido al agotamiento de los folículos, es un evento fisiológico en la vida de la mujer, no obstante se presenta acompañada de diversas modificaciones de tipo orgánico y psicológico, algunas de inicio inmediato y otras de instauración tardía, que en su conj unto constituyen el Síndrome Climatérico (5-7).

Los conocimientos anatómicos, fisiopatológicos, clínicos y epidemiológicos sobre el climaterio femenino son en extremo de suma importancia para el personal de salud y también para la población en general.

En especial, para la profesional de la salud mujer; quién formará parte de la población que requerirá atención sanitaria (1).

Existen diversos trabajos sobre conocimientos y actitudes sobre climaterio y menopausia realizados en diversas latitudes realizadas con mujeres de diferente edad y diferente estrato social (8-11) más no hemos encontrado en nuestro medio ningún estudio que evalúe a los profesionales de la salud, 
en especial al Obstetra, sobre cuánto conoce del climaterio femenino.

Nuestro estudio, realizado en diferentes regiones de la costa y sierra del Perú; nos muestra que existe la necesidad de tener a profesionales debidamente capacitados para atender a esta población femenina que cada vez acude en mayor número por un consejo o una terapia diferenciada durante el climaterio.

En este trabajo se encuestó a una población de profesionales de la salud que tenían características muy similares en su formación académica y estrato social, con la característica uniforme de tener interés por capacitarse y mejorar su experticia en el manejo del climaterio, por lo tanto; fue un grupo motivado y diferenciado.

Los resultados, nos permiten conocer que las Obstetras a pesar de recibir clases en el pregrado, desconocen el manejo integral del climaterio. Esto significa, que confunden términos y no son capaces de reconocer características clínicas asociadas al climaterio $(12,13)$. Lo que se hace evidente, al no encontrar diferencia significativa al comparar tiempo de egreso.

Otro aspecto importante a destacar, es que sólo el grupo que trabaja en EsSalud; en alguna oportunidad recibió capacitación sobre el manejo del climaterio pero fue insuficiente. Existiendo diversidad de opinión y poco criterio para decidir por el tratamiento más adecuado.

Siendo las más j óvenes y con poco tiempo de egreso las más dispuestas y convencidas del beneficio de la terapia hormonal. Existiendo mayor predisposición en las de más de 10 años de egresadas por recomendar tratamiento no hormonal, lo que en cierta medida puede ser el reflejo de la controversia surgida después del 2002, ante la publicación del estudio WHI (14).

Todos los encuestados reconocieron la necesidad de ofrecer Consejería de calidad y valoraron el estilo de vida saludable como la mejor estrategia de promoción y prevención de la salud (15-17). Siendo la Psicoprofilaxis del climaterio una de las alternativas para mejorar la calidad de vida de la población mayor de 40 años.

Una limitación del presente estudio es el hecho de no poder evaluar el impacto del nivel socioeconómico de la paciente, ni el tiempo de consulta que se le dedica durante la consulta ambulatoria por una molestia en su climaterio.

Consideramos de necesidad el desarrollo de un Plan Nacional de Manejo del Climaterio, pues cada vez habrá un mayor número de mujeres que acudirán por una atención especializada, al llegar la menopausia (18).

En conclusión, no hubo diferencia significativa entre el tiempo de egreso y el nivel de conocimiento, pero sí en la disposición y manejo de terapia hormonal por las más jóvenes.

Siendo muy significativo el nivel de falta de conocimiento tanto en el profesional de la costa como de la sierra. El $100 \%$ estuvo de acuerdo en la necesidad de capacitarse en esta etapa de la vida.

\section{Fuente de Financiamiento}

El presente trabajo fue financiado parcialmente por la Sociedad Peruana de Psicoprofilaxis Obstétrica (ASPPO) y por el autor.

\section{Conflictos de interés}

El autor declara no tener ningún conflicto de interés.

\section{REFERENCIAS BIBLIOGRÁFICAS}

1. INEI, Perú. Estimaciones y proyecciones de población, 1950-2050. Boletín de Análisis Demográfico 2013; 36.

2. Paredes N. En: Aspectos Demográficos. Hacia una nueva primavera de la mujer después de los 40. Cimagraf. Segunda edición; LimaPerú. 2008:15-18. 
3. Paredes N. Fitoestrógenos: ¿La alternativa de los estrógenos?.Rev Per Climat 2001; 3:7-10.

4. Morales S. Preparación integral para el parto y etapas del ciclo de vida. Sílabo EPO-USMP 2012-II.

5. Blumel JE, Roncagliolo ME, Gramegna G, Tacla X, Sepulveda H. Prevalencia de síntomas psíquicos y vasomotores en diferentes periodos del Climaterio. RevChilObstetGinecol 1997; 62:412-8.

6. Paredes N, Climaterio y Metabolismo del calcio. RevMed Moderna 1993; 1:20-25.

7. Baker A; Simpson S; Dawson D. Sleep disruption and mood changes associated with menopause. J Psychosom Res 1997; 43: 359-69.

8. Pacheco J, Salvador J. Conocimientos y actitudes de la mujer peruana sobre climaterio y menopausia. Rev Per Obstet y Ginecol 1997; 43(1): 33-38.

9. Távara L, Sacsa D, Molina N, Espinoza K, Inguil R. Conocimiento y uso de la hormonoterapia de reemplazo entre mujeres climatéricas y de clase media en Lima Metropolitana. Rev Per Obstet y Ginecol 1998; 44:186-91.

10. Monterrosa A. Barrios J, Bello A.

Conocimientos y creencias sobre menopausia en mujeres mayores de 40 años de edad, naturales del Palenque de San Basilio.

Bolívar-Colombia. http://encolombia.com conocimientos_meno5-1.htm.

11. Vera A, Rentería S. Conceptos y creencias sobre menopausia. Estudio en 240 mujeres distribuidas en dos grupos de edades. Rev Col Menop 1996; 2(1):51-56.

12. Brown WJ, Mishra GD, Dobson A. Changes in physical symptoms during the menopause transition. Int J Behav Med 2002; 9:53-67.
13. Hardy R, Kuh D. Change in psychological and vasomotor symptom reporting during the menopause.SocSci Med 2002; 55:1975-88.

14. WHI. Risks and Benefits of Estrogen Plus Progestin in Healthy Postmenopausal WomenPrincipal Results From the Women's Health Initiative Randomized Controlled Trial. JAMA. 2002;288(3):321-333.

15. Elavsky S,McAuley E. Personality, menopausal symptoms, and physical activity outcomes in middle-aged women. Personality and Individual Differences 2009;46(2):123-128.

16. Coutinho de Azevedo A, Baptista F.

Relationship between physical activity and menopausal symptoms. Rev Bras AtividadeFisSaude 2011;16(2):144-149.

17. Paredes N. Beneficio del ejercicio aeróbico sobre los síntomas vasomotores de pacientes postmenopáusicas. Horiz Med 2013; 13(3):1524.

18. Blumel JE, Chedraui P, Baron G, et al. Collaborative Group for Research of the Climacteric in Latin America (REDLINC). Sexual dysfunction in middle-aged women: a multicenter Latin American study using the Female Sexual Function Index. Menopause 2009; 16:1139-48.

\section{Correspondencia:}

Nieves Bedón Cortéz

Dirección: Av. JP Oeste 555-1501, Lima-Perú

Teléfono: (511) 6474925

Correo electrónico: asppo@asppo.org 